\title{
Diversity Analysis of Analog Network Coding with Multi-User Interferences
}

\author{
Wei Guan and K. J. Ray Liu
}

\begin{abstract}
Network coding can improve the bandwidth efficiency by letting multiple users share the relay channels. However, the multi-user interferences (MUI) at the intended receiver may degrade the diversity performances. So in this work, we study several diversity strategies for the multiple-access relay channel and analyze the impact of MUI on the diversity performances. Both the variable gain relaying (VGR) and the fixed gain relaying (FGR) are considered, where the relay nodes are subject to instantaneous power constraints and long-term power constraints, respectively. We first develop a Min-Max relay selection strategy and prove that full diversity can be achieved. Next, the distributed space-time block coding (DSTBC) and the diagonal distributed space-time coding (DDSTC) are studied. For DSTBC, we show that full diversity can be achieved by FGR, but the diversity of VGR is upper bounded by $\min (L+1, K+1)$, where $L$ and $K$ are the number of relays and users, respectively. For single-user systems, we then develop a selective DSTBC-VGR scheme to recover the diversity loss by adaptively allocating the relay power. Finally for DDSTC, we show that both FGR and VGR can achieve full diversity, and the optimum code design criterion is to maximize the minimum product distance.
\end{abstract}

Index Terms-Network coding, wireless relaying, interference, diversity.

\section{INTRODUCTION}

$\mathbf{C}$ OOPERATIVE communications have gained great attentions recently due to the potential to provide spatial diversity, extend transmission range and save transmitted power [1]. The two most popular cooperation protocols are amplifyand-forward (AF) and decode-and-forward (DF) [2]. For DF protocol, the random decoding error at the relay node may propagate to the intended receiver, which will severely degrade the diversity performances [3]-[5]. For AF protocol, early literatures [2][6] have shown that full diversity can always be achieved for the single-user systems.

The single-user relay channel has been widely studied in the community. For example, the outage probability is obtained in [7] for the multi-relay networks using orthogonal channels. Although full diversity can be achieved, the loss in terms of bandwidth efficiency increases linearly with the number of relays. Many multi-node cooperation schemes have been proposed to address this issue. For example, distributed beamforming [8][9] can achieve full diversity by properly adjusting the relay power and rotation coefficients. Alternatively, if only the best relay node is selected to forward data each time,

Manuscript received December 17, 2011; revised April 12 and August 4, 2012; accepted October 28, 2012. The associate editor coordinating the review of this paper and approving it for publication was Y. Jing.

The authors are with the Department of Electrical and Computer Engineering, University of Maryland, College Park, MD 20742 (e-mail: \{wguan, kjrliu\}@umd.edu).

Digital Object Identifier 10.1109/TWC.2012.121712.112232 full diversity is still achievable due to order statistics [10]. Another way to realize multi-node cooperation is through distributed space-time coding (DSTC), where the relay nodes perform linear coding on the received signals. The distributed space-time block coding (DSTBC) and the diagonal distributed space-time coding (DDSTC) have been studied in [11] and [12], respectively, and some code design examples can be found in [13][14].

In practice, the user devices usually cannot transmit and receive on the same channel due to hardware constraints. So the conventional half-duplex relaying protocols will suffer some rate loss, as the transmission of one packet needs two orthogonal channels. One way to improve the bandwidth efficiency is to let multiple users share the relay channels through network coding, and such idea has been widely applied in the two-way relay channel (TWRC) and the multiple-access relay channel (MARC). One critical issue needs to be addressed here is the resulting multi-user interferences (MUI) at the intended receiver. For TWRC, the end node can efficiently mitigate the MUI by subtracting the self-interferences from the received signals [15][16]. On the contrary, for MARC the MUI always exists and may degrade the diversity performances. In [17], the authors demonstrate that the two-user single-relay MARC can achieve the optimum diversity and multiplexing tradeoff (DMT) at high multiplexing gains, while at low multiplexing gains the DMT is such as if there is no MUI. Later in [18], the optimum DSTC schemes to achieve the above DMT bound are constructed in an algebraic way. For the MARC with two users and multiple parallel relays, [19] derives the optimum rate regions by imposing the sum-power constraints on the relay array. A distributed relay selection strategy is proposed in [20], where the authors analyze the resulting outage capacity and ergodic capacity. In [21], a distributed beamforming strategy is proposed to maximize the achievable sum-rate when the input noises are correlated. Likewise, [22] develops two relaying schemes to minimize the mean squared error at the destinations, where the relays either cooperate globally or just leverage the local signals. In [23], the beamforming design when only the quantized channel state information (CSI) is available at the relays is studied, and a generalized diversity measure is introduced to study the impact of MUI on the diversity performances.

We remark that most of the above literatures [17]-[22] are from an information-theoretic view, which ideally assumes that the channel input is Gaussian and the codeword is infinitely long. In practice, to know the error performances of the practical coding and modulation schemes are usually more important to evaluate the practical network performances. 
Although [23] derives the diversity bound of the distributed beamforming strategy with quantized feedback, it focuses only on the instantaneous relay power constraints; besides, when it is hard to obtain the real-time CSI, how to achieve spatial diversity is still unclear.

So in this work, we provide a comprehensive study on the error performances of the multi-user network-coded systems using analog relaying transmission, where the $K$ users are sending data to the common destination with the help of $L$ parallel relays, as shown in Fig. 1. The two basic issues we are going to address involve how to achieve spatial diversity when there exists MUI and what is the impact of MUI on the diversity performances. Depending on the relay power constraints, we investigate both the variable gain relaying (VGR) and the fixed gain relaying (FGR). We first study the single-relay networks, and show that full diversity can be achieved regardless of MUI. However, an logarithmic term will appear in the error rate expression and incur diversity loss at modest signal-to-noise ratios (SNR). Several relaying schemes to achieve distributed spatial diversity when there are multiple relays are then explored. We first propose a relay selection strategy based on the principle of minimizing the maximum pairwise error probability (PEP) and prove that full diversity can be achieved. Next, two DSTC schemes are studied. For DSTBC, we show that DSTBC-FGR can always achieve full diversity, whereas the diversity of DSTBC-VGR is upper bounded by $\min (L+1, K+1)$. As the diversity of the single-user DSTBC-VGR systems is limited by 2 , we develop an adaptive relay power allocation scheme that can recover the diversity loss. Finally for DDSTC, we show that both VGR and FGR can achieve full diversity, and the optimum code design criterion is to maximize the minimum product distance.

The rest of this paper is organized as follows: in Section II, we first study the single-relay systems. A relay selection strategy is then developed in Section III. We proceed to study the DSTBC and DDSTC schemes in Section IV and Section $\mathrm{V}$, respectively. Finally we present some simulation results in Section VI, and some conclusions are drawn in Section VII.

Notations: $|\cdot|,(\cdot)^{T}$ and $(\cdot)^{H}$ stand for absolute value, transpose and conjugate transpose, respectively. The boldface lowercase letter a and the boldface uppercase letter $\mathbf{A}$ represent vector in column form and matrix, respectively. $\|\mathbf{a}\|$ and $\operatorname{det} \mathbf{A}$ denote the Euclidean norm of a vector $\mathbf{a}$ and the determinant of a square matrix $\mathbf{A}$, respectively. $\mathbb{Z}$ and $\mathbb{C}$ stand for the set of integers and the set of complex numbers, respectively. We shall use the abbreviation i.i.d. for independent and identically distributed, and denote $Z \sim \mathcal{C N}\left(\mu, \sigma^{2}\right)$ as a circularly symmetric complex Gaussian random variable with the real part and the imaginary part being i.i.d. $\mathcal{N}\left(\mu, \frac{\sigma^{2}}{2}\right)$. The probability of an event $\mathcal{A}$ is denoted by $\operatorname{Pr}(\mathcal{A})$. The cumulative distribution function (CDF) and the probability density function (PDF) of a random variable $Z$ are denoted by $F_{Z}(z)$ and $f_{Z}(z)$, respectively. We define the Q-function as $Q(x)=\frac{1}{\sqrt{2 \pi}} \int_{x}^{\infty} e^{-\frac{t^{2}}{2}} d t$. Finally, we say $h(x)=\mathrm{O}(g(x))$ if $\lim \sup _{x \rightarrow \infty} \frac{h(x)}{g(x)}<\infty$.

\section{Multi-User Single-Relay Systems}

In this section, we first study the error performances of the multi-user single-relay systems. The analytical results obtained

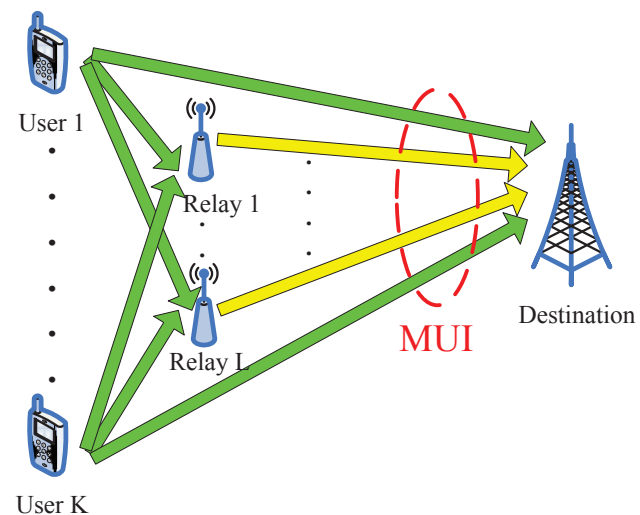

Fig. 1. Diagram of a general multi-user multi-relay network.

here will be used repeatedly in later sections when we consider the multi-relay networks.

\section{A. System Model}

Consider a multiple-access channel with $K$ users sending data to a single destination with the help of a single relay node, as shown in Fig. 1 with $L=1$. Let $f_{k} \sim \mathcal{C N}(0,1)$ be the channel coefficient from the $k$ th user to the relay, $h_{k} \sim \mathcal{C N}(0,1)$ be the channel coefficient from the $k$ th user to the destination, and $g \sim \mathcal{C N}(0,1)$ be the channel coefficient from the relay to the destination, respectively. All the channel coefficients are independent, and the additive noises on different channels are also independently distributed as $\mathcal{C N}(0,1)$. Without loss of generality, throughout this work we focus only on the symmetric networks, where all the user-relay channels have the same path-loss coefficient $\lambda_{s r}$, and all the user-destination channels have the same pathloss coefficient $\lambda_{s d}$. The path-loss coefficient of the relaydestination channel is denoted by $\lambda_{r d}$. We remark that such assumption is just to simplify the notations, and our analysis can be easily extended to any asymmetric networks. As will be clear later, these path-loss coefficients are only related to the coding gain but have nothing to do with the diversity order, which is the main concern of this work.

Due to the half-duplex relaying constraints, the whole data transmission takes place in two phases. In the first phase, all the users broadcast their data simultaneously, and the received signal at the relay and at the destination can be respectively represented as ${ }^{1}$

$$
\left\{\begin{array}{l}
y_{s r}=\sqrt{P \lambda_{s r}} \sum_{k=1}^{K} f_{k} s_{k}+n_{s r}, \\
y_{s d}=\sqrt{P \lambda_{s d}} \sum_{k=1}^{K} h_{k} s_{k}+n_{s d} .
\end{array}\right.
$$

Here $P$ is the transmitted power; $n_{s d}$ and $n_{s r}$ are the additive noises; and $s_{k}$ is the transmitted symbol of the $k$ th user, which is picked from some constellation $\Omega$ with normalized power, i.e., $E\left|s_{k}\right|^{2}=1$. The transmitted signal of the relay node is $x_{r}=\sqrt{\alpha P} y_{s r}$, where $\alpha$ is the amplification factor

\footnotetext{
${ }^{1}$ In this work, we assume all the transmitters are perfectly synchronized. The effect of synchronization errors is beyond the scope of this work.
} 
to normalize the relay power. In this work, we consider two different ways to normalize the relay power. For VGR, the amplification factor is chosen in such a way that the relay power is limited to $P$ at any time instant, i.e., $E\left(\left|x_{r}\right|^{2} \mid \mathbf{f}\right)=P$ with $\mathbf{f}=\left(f_{1}, f_{2}, \cdots, f_{K}\right)^{T}$. This requires the relay node to adjust the amplification factor according to the real-time channel conditions, thus $\alpha_{V G R}$ keeps changing all the time and is given by

$$
\alpha_{V G R}=\frac{1}{P \lambda_{s r} \sum_{k=1}^{K}\left|f_{k}\right|^{2}+1} .
$$

Alternatively, the relay node could also use a constant amplification factor such that the average relay power is normalized to $P$ in the long run, i.e., $E\left|x_{r}\right|^{2}=P$, which is referred to as FGR. The resulting amplification factor $\alpha_{F G R}$ is given by

$$
\alpha_{F G R}=\frac{1}{K P \lambda_{s r}+1} .
$$

Note that in this scheme, the amplification factor $\alpha_{F G R}$ is a constant depending only on the second-order statistics of channel distributions, so the relay node needs not to know the instantaneous channel conditions. It should also be pointed out that the relay power of FGR may momentarily exceed the maximum load of the power amplifier. However, such power saturation issue is not considered in this work to simplify the analysis.

After proper power scaling, the relay node then forwards the amplified signal to the destination in the second phase. The received signal is

$$
y_{r d}=\sqrt{\lambda_{r d}} g x_{r}+n_{r d}=\sqrt{\alpha P^{2} \lambda_{s r} \lambda_{r d}} g \sum_{k=1}^{K} f_{k} s_{k}+\tilde{n}_{r d},
$$

where $\tilde{n}_{r d} \triangleq \sqrt{\alpha P \lambda_{r d}} g n_{s r}+n_{r d} \sim \mathcal{C N}\left(0, \alpha P \lambda_{r d}|g|^{2}+1\right)$ is the equivalent additive noise. Upon observing the signals $y_{s d}$ and $y_{r d}$, the destination performs maximum-likelihood detection (MLD) to jointly decode the $K$ user symbols as

$$
\begin{aligned}
\mathbf{s}_{d}=\arg \min _{\hat{s}_{k} \in \Omega}\left|y_{s d}-\sqrt{P \lambda_{s d}} \sum_{k=1}^{K} h_{k} \hat{s}_{k}\right|^{2} \\
+\frac{\left|y_{r d}-\sqrt{\alpha P^{2} \lambda_{s r} \lambda_{r d}} g \sum_{k=1}^{K} f_{k} \hat{s}_{k}\right|^{2}}{\alpha P \lambda_{r d}|g|^{2}+1},
\end{aligned}
$$

where $\mathbf{s}_{d}=\left(s_{d, 1}, s_{d, 2}, \cdots, s_{d, K}\right)^{T}$ is the decoded symbol vector, and different channel noises are assumed to be independent.

\section{B. Performance Analysis}

In this subsection, we study the PEPs of the aforementioned systems. PEP is defined as the probability that a transmitted symbol $\mathbf{x}$ is mistaken by a different symbol $\hat{\mathbf{x}}$, which is well known to be a tight bound on the error rates [1]. As we shall see later, the asymptotic PEPs at high SNRs can be generally expressed as $\mathrm{O}\left(\frac{(\log P)^{d_{2}}}{P^{d_{1}}}\right)$, i.e., the diversity gain is $d_{1}-d_{2} \frac{\log \log P}{\log P}$. Although the impact of the logarithmic term vanishes at extremely high SNRs, i.e., $\lim _{P \rightarrow \infty} \frac{\log \log P}{\log P}=0$, it would incur some diversity loss at modest SNRs. For example, $\frac{\log \log P}{\log P} \geq 0.28$ when $P \leq 30 \mathrm{~dB}$. To capture the effect of this logarithmic term, we follow the convention of [23] and define the generalized diversity gain as a tuple $\left(d_{1},-d_{2}\right)$, where $d_{1}$ is the dominant diversity gain that characterizes the asymptotic behaviors of the error rates at extremely high SNRs, and $d_{2}$ determines the extent of performance loss at modest SNRs. We say full diversity is achieved when $d_{1}=L+1$ regardless of the value of $d_{2}$, where $L$ is the number of relays in the networks and the additional diversity gain comes from the direct source-destination links.

According to (5), the PEP of mistaking $\mathbf{s}$ by $\hat{\mathbf{s}}$ is given by

$$
\begin{aligned}
\operatorname{Pr}(\mathbf{s} \rightarrow \hat{\mathbf{s}}) & =E\left[Q\left(\sqrt{2\left(W_{d}+W_{r}\right)}\right)\right] \\
& \leq E\left[\exp \left(-\left(W_{d}+W_{r}\right)\right)\right],
\end{aligned}
$$

where $W_{d}=\frac{1}{4} P \lambda_{s d}\left|\mathbf{h}^{T} \Delta \mathbf{s}\right|^{2}, W_{r}=\frac{\alpha P^{2} \lambda_{s r} \lambda_{r d}|g|^{2}\left|\mathbf{f}^{T} \Delta \mathbf{s}\right|^{2}}{4\left(\alpha P \lambda_{r d}|g|^{2}+1\right)}$, $\mathbf{h}=\left(h_{1}, h_{2}, \cdots, h_{K}\right)^{T}, \Delta \mathbf{s}=\mathbf{s}-\hat{\mathbf{s}}$, and we have applied Chernoff bound [1] in the inequality. As $W_{d}$ follows the exponential distribution, we have

$$
E\left[\exp \left(-W_{d}\right)\right]=\frac{4}{P \lambda_{s d}\|\Delta \mathbf{s}\|^{2}+4} \stackrel{P \rightarrow \infty}{\approx} \frac{4}{\lambda_{s d}\|\Delta \mathbf{s}\|^{2}} P^{-1} .
$$

To evaluate the expectation of the second term in (6), we first prove the following lemma.

Lemma 1: Let $W_{1}=\frac{a b X Y}{a X+b Y+c Z+1}$ and $W_{2}=\frac{a b X Y}{a X+c}$, where $a, b$ and $c$ are constants, $X$ and $Y$ are independent exponential random variables with unit mean, and $Z$ is independent of $X$ and $Y$ and has the gamma distribution $f(z)=\frac{1}{\Gamma(N)} z^{N-1} e^{-z}$, then for $w \geq 0$ the CDF of $W_{1}$ and $W_{2}$ are respectively given by

$$
\begin{aligned}
& F_{W_{1}}(w) \leq 1+\frac{N c w}{a b} \exp \left(-\frac{a+b-c}{a b} w\right) E_{1}\left(\frac{c w}{a b}\right) \\
&-\exp \left(-\frac{a+b}{a b} w\right) 2 \sqrt{\frac{w+w^{2}}{a b}} K_{1}\left(2 \sqrt{\frac{w+w^{2}}{a b}}\right) \\
& w \ll 1 \frac{N c+1}{a b} w \log \frac{1}{w}, \\
& F_{W_{2}}(w)=1-\exp \left(-\frac{w}{b}\right) \sqrt{\frac{4 w c}{a b}} K_{1}\left(\sqrt{\frac{4 w c}{a b}}\right) \\
& w \ll 1 \frac{c}{a b} w \log \frac{1}{w},
\end{aligned}
$$

where $E_{1}(x)=\int_{x}^{\infty} \frac{e^{-t}}{t} d t$ is the exponential integral function [24, 5.1.1], and $K_{1}(x)$ is the first-order modified Bessel function of the second kind [24, 9.6.1].

Proof: See Appendix A.

Now we proceed to study the PEPs of FGR and VGR, respectively. For FGR, after plugging (3) in $W_{r}$, we have $W_{r, F G R}=\frac{P^{2} \lambda_{s r} \lambda_{r d}|g|^{2}\left|\mathbf{f}^{T} \Delta \mathbf{s}\right|^{2}}{4\left(P \lambda_{r d}|g|^{2}+K P \lambda_{s r}+1\right)}$. According to Lemma 1, the CDF of $W_{r, F G R}$ can be obtained by substituting $a_{1}=P \lambda_{r d}$, $b_{1}=\frac{1}{4} P \lambda_{s r}\|\Delta \mathbf{s}\|^{2}$, and $c_{1}=K P \lambda_{s r}+1$ in (9). Then we can derive (10) shown on the top of the next page, where we use $[25,6.643 .3],[24,13.1 .33]$ and $[24,13.2 .5]$ in $(a)$, and the inequality $E_{1}(z)<e^{-z} \log \left(1+\frac{1}{z}\right)[24,5.1 .20]$ in $(b)$. 


$$
\begin{aligned}
E\left[\exp \left(-W_{r, F G R}\right)\right] & \stackrel{(a)}{=} \frac{1}{b_{1}+1}+\frac{b_{1} c_{1}}{a_{1}\left(b_{1}+1\right)^{2}} \exp \left(\frac{c_{1}}{a_{1}\left(b_{1}+1\right)}\right) E_{1}\left(\frac{c_{1}}{a_{1}\left(b_{1}+1\right)}\right) \\
& \stackrel{(b)}{\leq} \frac{1}{b_{1}+1}+\frac{b_{1} c_{1}}{a_{1}\left(b_{1}+1\right)^{2}} \log \left(1+\frac{a_{1}\left(b_{1}+1\right)}{c_{1}}\right) \stackrel{P \rightarrow \infty}{\approx} \frac{4 K}{\lambda_{r d}\|\Delta \mathbf{s}\|^{2}} \frac{\log P}{P},
\end{aligned}
$$

$$
\begin{aligned}
& E\left[\exp \left(-W_{r, V G R}\right)\right] \stackrel{(a)}{\leq} 1-\frac{\|\Delta \mathbf{s}\|^{2}}{4} \exp \left(-\frac{1}{\sqrt{a_{2} b_{2}}}\right)\left(\frac{\|\Delta \mathbf{s}\|^{2}}{4}+\frac{\left(\sqrt{a_{2}}+\sqrt{b_{2}}\right)^{2}}{a_{2} b_{2}}\right)^{-1} \\
& \quad+\frac{(K-1) a_{2}\|\Delta \mathbf{s}\|^{2}}{4}\left(\frac{16}{\|\Delta \mathbf{s}\|^{4} a_{2}^{2}} \log \left(1+\frac{\|\Delta \mathbf{s}\|^{2} a_{2}}{4}\right)-\frac{1}{\frac{\|\Delta \mathbf{s}\|^{2} a_{2}}{4}\left(1+\frac{\|\Delta \mathbf{s}\|^{2} a_{2}}{4}\right)}\right) \stackrel{P \rightarrow \infty}{\approx} \frac{4(K-1)}{\|\Delta \mathbf{s}\|^{2} \lambda_{r d}} \frac{\log P}{P},
\end{aligned}
$$

Combining (7) with (10) leads to

$$
\begin{aligned}
& \operatorname{Pr}(\mathbf{s} \rightarrow \hat{\mathbf{s}} \mid \mathrm{FGR}) \stackrel{P}{\stackrel{\sim}{\approx}} \frac{16 K}{\lambda_{r d} \lambda_{s d}\|\Delta \mathbf{s}\|^{4}} \frac{\log P}{P^{2}} \\
& \leq \frac{16 K}{\lambda_{r d} \lambda_{s d} d_{\text {min }}^{4}} \frac{\log P}{P^{2}},
\end{aligned}
$$

where $d_{\min }=\min _{s, \hat{s} \in \Omega, s \neq \hat{s}}|s-\hat{s}|$ is the minimum distance of any two distinct points in the set $\Omega$.

Next we study the PEPs of VGR. After plugging (2) in $W_{r}$, we have $W_{r, V G R}=\frac{P^{2} \lambda_{s r} \lambda_{r d}|g|^{2}\left|\mathbf{f}^{T} \Delta \mathbf{s}\right|^{2}}{4\left(P \lambda_{r d}|g|^{2}+P \lambda_{s r} \sum_{k=1}^{K}\left|f_{k}\right|^{2}+1\right)}$. For any error vector $\Delta \mathbf{s} \neq 0$, we can always find a $K \times K$ unitary matrix $\mathbf{U}$ with the first row being $\frac{\Delta \mathbf{s}^{T}}{\|\Delta \mathbf{s}\|}$. Define a new vector $\tilde{\mathbf{f}}=\mathbf{U f}$, then we have $W_{r, V G R}=\frac{\|\Delta \mathbf{s}\|^{2}}{4} \tilde{W}_{r, V G R}$ with $\tilde{W}_{r, V G R}=\frac{P^{2} \lambda_{s r} \lambda_{r d}|g|^{2}\left|\tilde{f}_{1}\right|^{2}}{P \lambda_{r d}|g|^{2}+P \lambda_{s r}\left|\tilde{f}_{1}\right|^{2}+P \lambda_{s r} \sum_{k=2}^{K}\left|\tilde{f}_{k}\right|^{2}+1}$. As $\tilde{\mathbf{f}} \sim$ $\mathcal{C N}(\mathbf{0}, \mathbf{I}),\left|\tilde{f}_{1}\right|^{2}$ is independent of $\sum_{k=2}^{K}\left|\tilde{f}_{k}\right|^{2}$, which has the gamma distribution. According to Lemma 1, the CDF of $\tilde{W}_{r, V G R}$ can be obtained after plugging $a_{2}=P \lambda_{r d}, b_{2}=$ $c_{2}=P \lambda_{s r}$ and $N=K-1$ in (8). Then we can derive (12) shown on the top of this page, where we use [25, 6.227.1] and the inequality $x K_{1}(x) \geq \exp (-x)$ [23] in (a). Combining (7) with (12) leads to

$$
\begin{aligned}
& \operatorname{Pr}(\mathbf{s} \rightarrow \hat{\mathbf{s}} \mid \mathrm{VGR}) \stackrel{P}{\stackrel{\sim}{\approx}} \frac{16(K-1)}{\lambda_{r d} \lambda_{s d}\|\Delta \mathbf{s}\|^{4}} \frac{\log P}{P^{2}} \\
& \leq \frac{16(K-1)}{\lambda_{r d} \lambda_{s d} d_{\text {min }}^{4}} \frac{\log P}{P^{2}}
\end{aligned}
$$

\section{Discussions}

It is observed that both FGR and VGR achieve the diversity gain $(2,-1)$ when there exists MUI (i.e., $K>1$ ). Recall that for the conventional single-user analog relaying networks, the diversity gain is $d_{V G R}=(2,0)$ [2][6] and $d_{F G R}=(2,-1)$ [11][12], respectively. Although all these schemes can achieve full diversity, only the singleuser VGR does not suffer any logarithmic-term loss at modest SNRs. To explain this phenomenon, let us revisit the relay signal model (4), where the signal component of the $k$ th user is given by $\sqrt{\alpha P^{2} \lambda_{s r} \lambda_{r d}} g f_{k} s_{k}$. For the singleuser VGR, the amplification factor is given by $\alpha_{V G R}=$
$\left(P \lambda_{s r}\left|f_{k}\right|^{2}+1\right)^{-1} \stackrel{P \rightarrow \infty}{\approx} \frac{1}{P \lambda_{s r}\left|f_{k}\right|^{2}}$, where the approximation holds with probability 1 . Consequently, the signal component becomes $h_{e q, k} s_{k}$ at high SNRs, and the equivalent channel $h_{e q, k} \triangleq \sqrt{P \lambda_{r d}} g e^{j \varphi\left(f_{k}\right)}$ still follows Rayleigh fading, where $\varphi\left(f_{k}\right)$ is the phase of $f_{k}$. On the contrary, for all the other cases the effective channel is proportional to $f_{k} g$, which follows double-Rayleigh fading [26] and induces the logarithmic term in the expressions of PEPs.

By comparing (11) and (13), we also observe that increasing the user number $K$ beyond 2 would not degrade the diversity performances further. However, since the dominant PEPs are proportional to $K$, there is some linear loss of coding gain as the number of users increases. Relatively speaking, VGR performs slightly better than FGR. This is because the instantaneous output power at the relay node is always normalized to $P$ in VGR, which helps to mitigate the extent of channel fading. Finally, it is also noteworthy that the dominant PEPs are inversely proportional to $\lambda_{r d}$ but is independent of $\lambda_{s r}$. This is because the received signal power of $y_{s r}$ is approximately proportional to $\lambda_{s r}$, so the pathloss effects of source-relay channels would be counteracted during power normalization at the relay node. Consequently, the quality of relay-destination channel dominates the overall error performances, and the best relay position should be closer to the destination.

\section{Relay Selection StRategy}

From now on, we consider the more general networks with multiple relays. The design objective is to achieve higher spatial diversity gain. In this section, we first develop a relay selection strategy based on the Min-Max criterion. More sophisticated DSTC schemes will be discussed in later sections.

The system model is a natural extension of the single-relay model discussed in the last section. Suppose there are now $L$ parallel relays. Let $f_{k l}$ be the channel coefficient from the $k$ th user to the $l$ th relay, and $g_{l}$ be the channel from the $l$ th relay to the destination. We still consider the symmetric networks, where all the user-relay channels have the same path-loss coefficient $\lambda_{s r}$, and all the relay-destination channels have the same path-loss coefficient $\lambda_{r d}$. As there are multiple parallel relays now, the relaying strategy becomes much more flexible. An intuitive scheme is to let each relay node forwards the uncoded data one after another during the second phase, and the destination then performs MLD by constructively 
combining all the received signals. Although this scheme does achieve full diversity, the bandwidth efficiency is really low, as $L$ independent channels are required for orthogonal relaying.

To address this issue, we propose to select only one good relay (i.e., the $q$ th relay) each time to help forward the source messages. The resulting signal model is basically the same as that in the single-relay networks after properly modifying the subscripts in (1a) and (4). To be specific, all the users still broadcast concurrently in the first phase. The received signal at the $q$ th relay is $y_{s r_{q}}=\sqrt{P \lambda_{s r}} \sum_{k=1}^{K} f_{k q} s_{k}+n_{s r_{q}}$, and the received signal at the destination is still given by (1b). Then in the second phase, the $q$ th relay will amplify and forward the data using either $\alpha_{q, V G R}=\left(P \lambda_{s r} \sum_{k=1}^{K}\left|f_{k q}\right|^{2}+1\right)^{-1}$ or $\alpha_{q, F G R}=\left(K P G \lambda_{s r}+1\right)^{-1}$. Note that $\alpha_{q, F G R}$ is slightly different from (3) after introducing the additional factor $G$. This is because after relay selection, the incoming channels are generally under very good conditions and the channel distributions would greatly change due to order statistics. As it is very hard to obtain the exact distribution functions, we simply assume that $\sum_{k=1}^{K} E\left|f_{k, q}\right|^{2}=K G$, where $G(>1)$ is a bounded constant that can be obtained using computer simulations, and this factor would not influence the diversity performances. The received signal at the destination during the second phase is $y_{r_{q} d}=\sqrt{\alpha P^{2} \lambda_{s r} \lambda_{r d}} g_{q} \sum_{k=1}^{K} f_{k q} s_{k}+\tilde{n}_{r_{q} d}$, where $\tilde{n}_{r_{q} d} \sim \mathcal{C N}\left(0, \alpha P \lambda_{r d}\left|g_{q}\right|^{2}+1\right)$. Finally, the MLD similar to (5) is performed to decode all the user symbols based on the observations $y_{s d}$ and $y_{r_{q} d}$, and the conditional PEP is given by

$$
\begin{aligned}
\operatorname{Pr}\left(\mathbf{s} \rightarrow \hat{\mathbf{s}} \mid \mathbf{h}, \mathbf{f}_{s q}, g_{q}\right) & =Q\left(\sqrt{2\left(W_{d}+W_{r_{q}}(\Delta \mathbf{s})\right)}\right) \\
& \leq \exp \left(-W_{d}-W_{r_{q}}(\Delta \mathbf{s})\right),
\end{aligned}
$$

where $\quad W_{d}=\frac{1}{4} P \lambda_{s d}\left|\mathbf{h}^{T} \Delta \mathbf{s}\right|^{2}, \quad W_{r_{q}}(\Delta \mathbf{s})=$ $\frac{\alpha P^{2} \lambda_{s r} \lambda_{r d}\left|g_{q}\right|^{2}\left|\mathbf{f}_{s q}^{T} \Delta \mathbf{s}\right|^{2}}{4\left(\alpha P \lambda_{r d}\left|g_{q}\right|^{2}+1\right)}$ and $\mathbf{f}_{s q}=\left(f_{1 q}, f_{2 q}, \cdots, f_{K q}\right)^{T}$. It is observed that the quality of the $q$ th relay branch is uniquely characterized by $W_{r_{q}}(\Delta \mathbf{s})$, which itself depends on the error vector $\Delta \mathbf{s}$. As the real error probability is lower bounded by any PEP, we propose to select the relay branch that can minimize the maximum PEP. Since the exponential function is monotonically decreasing, the above Min-Max relay selection strategy can be equivalently formulated as

$$
\begin{aligned}
q & =\arg \min _{l=1,2, \cdots, L} \max _{\hat{\mathbf{s}} \neq \mathbf{s}} \operatorname{Pr}\left(\mathbf{s} \rightarrow \hat{\mathbf{s}} \mid \mathbf{h}, \mathbf{f}_{s l}, g_{l}\right) \\
& =\arg \max _{l=1,2, \cdots, L} \min _{\Delta \mathbf{s} \neq \mathbf{0}} W_{r_{l}}(\Delta \mathbf{s}) .
\end{aligned}
$$

We remark that the above Min-Max criterion is also independently studied in [23] for VGR without considering the direct link. In the following, we shall obtain the diversity performances of both VGR and FGR by using a much simpler approach.

Proposition 1: The diversity gain of both VGR and FGR using the Min-Max relay selection strategy (15) is $(L+1,-L)$.
Proof: Let $W_{r_{l}}^{*}=\min _{\Delta \mathbf{s} \neq \mathbf{0}} W_{r_{l}}(\Delta \mathbf{s})$ and $W_{r_{q}}^{*}=$ $\max _{l=1,2, \cdots, L} W_{r_{l}}^{*}$, then we have

$$
\begin{aligned}
F_{W_{r_{q}}(\Delta \mathbf{s})}(w) & \leq \operatorname{Pr}\left(W_{r_{q}}^{*} \leq w\right)=\prod_{l=1}^{L} \operatorname{Pr}\left(W_{r_{l}}^{*} \leq w\right) \\
& \leq \prod_{l=1}^{L} \sum_{\Delta \mathbf{s} \neq \mathbf{0}} F_{W_{r_{l}}(\Delta \mathbf{s})}(w) .
\end{aligned}
$$

For VGR, we have

$$
\begin{aligned}
W_{r_{l}, F G R}(\Delta \mathbf{s}) & =\frac{P^{2} \lambda_{s r} \lambda_{r d}\left|g_{l}\right|^{2}\left|\mathbf{f}_{s l}^{T} \Delta \mathbf{s}\right|^{2}}{4\left(P \lambda_{r d}\left|g_{l}\right|^{2}+K P G \lambda_{s r}+1\right)} \\
& \stackrel{P \rightarrow \infty}{\geq} \frac{P \lambda_{s r} \lambda_{r d}\left|g_{l}\right|^{2}\left|\mathbf{f}_{s l}^{T} \Delta \mathbf{s}\right|^{2}}{4\left(\lambda_{r d}\left|g_{l}\right|^{2}+K G \lambda_{s r}+1\right)} .
\end{aligned}
$$

The CDF of $W_{r_{l}, F G R}(\Delta \mathbf{s})$ is thus given by

$$
\begin{aligned}
& F_{W_{r_{l}, F G R}(\Delta \mathbf{s})}(w) \\
& \quad \stackrel{P \rightarrow \infty}{\leq} \operatorname{Pr}\left(\frac{\lambda_{s r} \lambda_{r d}\left|g_{l}\right|^{2}\left|\mathbf{f}_{s l}^{T} \Delta \mathbf{s}\right|^{2}}{4\left(\lambda_{r d}\left|g_{l}\right|^{2}+K G \lambda_{s r}+1\right)} \leq \frac{w}{P}\right) \\
& \quad \stackrel{P \rightarrow \infty}{\stackrel{c_{3}}{a_{3} b_{3}} \frac{w}{P} \log \left(\frac{P}{w}\right),}
\end{aligned}
$$

where $a_{3}=\lambda_{r d}, b_{3}=\frac{\lambda_{s r}\|\Delta \mathbf{s}\|^{2}}{4}, c_{3}=K G \lambda_{s r}+1$, and the approximation is due to (9). Likewise, for VGR we have

$$
\begin{aligned}
W_{r_{l}, V G R}(\Delta \mathbf{s}) & =\frac{P^{2} \lambda_{s r} \lambda_{r d}\left|g_{l}\right|^{2}\left|\mathbf{f}_{s l}^{T} \Delta \mathbf{s}\right|^{2}}{4\left(P \lambda_{r d}\left|g_{l}\right|^{2}+P \lambda_{s r} \sum_{k=1}^{K}\left|f_{k l}\right|^{2}+1\right)} \\
& \stackrel{P \rightarrow \infty}{\geq} \frac{P \lambda_{s r} \lambda_{r d}\left|g_{l}\right|^{2}\left|\mathbf{f}_{s l}^{T} \Delta \mathbf{s}\right|^{2}}{4\left(\lambda_{r d}\left|g_{l}\right|^{2}+\lambda_{s r} \sum_{k=1}^{K}\left|f_{k l}\right|^{2}+1\right)} .
\end{aligned}
$$

The CDF of $W_{r_{l}, V G R}(\Delta \mathbf{s})$ is given by

$$
\begin{aligned}
& F_{W_{r_{l}, V G R}(\Delta \mathbf{s})}(w) \\
& \stackrel{P \rightarrow \infty}{\leq} \operatorname{Pr}\left(\frac{\lambda_{s r} \lambda_{r d}\left|g_{l}\right|^{2}\left|\mathbf{f}_{s l}^{T} \Delta \mathbf{s}\right|^{2}}{4\left(\lambda_{r d}\left|g_{l}\right|^{2}+\lambda_{s r} \sum_{k=1}^{K}\left|f_{k l}\right|^{2}+1\right)} \leq \frac{w}{P}\right) \\
& \stackrel{P}{\underset{\sim}{*}} \frac{(K-1) c_{4}+1}{a_{4} b_{4}} \frac{w}{P} \log \left(\frac{P}{w}\right),
\end{aligned}
$$

where $a_{4}=\lambda_{r d}, b_{4}=\frac{\lambda_{s r}\|\Delta \mathbf{s}\|^{2}}{4}, c_{4}=\lambda_{s r}$, and we use the small value approximation in (8). Plugging (17) and (18) back into (16) leads to $F_{W_{r_{q}}(\Delta \mathbf{s})}(w) \stackrel{P \rightarrow \infty}{\leq} C\left[\frac{w}{P} \log \left(\frac{P}{w}\right)\right]^{L}$, where $C$ is some constant. Therefore, for both the VGR and FGR 
we can obtain

$$
\begin{aligned}
& E\left[\exp \left(-W_{r_{q}}(\Delta \mathbf{s})\right)\right] \\
& \stackrel{P \rightarrow \infty}{\leq} C P(-1)^{L} \int_{0}^{\infty} \exp (-P w) w^{L}(\log w)^{L} d w \\
& \left.\quad \stackrel{(a)}{=} C P(-1)^{L} \frac{\partial^{L}}{\partial w^{L}}\left\{P^{-w} \Gamma(w)\right\}\right|_{w=L+1} \\
& \quad=\left.C P(-1)^{L}\left\{\sum_{k=0}^{L}\left(\begin{array}{l}
L \\
k
\end{array}\right) \frac{\partial^{k} P^{-w}}{\partial w^{k}} \frac{\partial^{L-k} \Gamma(w)}{\partial w^{L-k}}\right\}\right|_{w=L+1} \\
& \left.\stackrel{P}{\approx} C P(-1)^{L}\left\{\frac{\partial^{L} P^{-w}}{\partial w^{L}} \Gamma(w)\right\}\right|_{w=L+1} \\
& \quad=C \Gamma(L+1) \frac{(\log P)^{L}}{P^{L}}
\end{aligned}
$$

where we use $[25,4.358 .5]$ in $(\mathrm{a})$, and $\Gamma(x)$ is the Gamma function [25, 8.310.1]. Combining the above result with (7) completes the proof.

\section{Distributed Space-Time Block Coding}

Although relay selection can achieve full diversity, it would induce noticeable loss of coding gain as each time there is only one relay node helping forward data. To fully exploit the spatial diversity, we investigate DSTBC in this section, where all the relay nodes participate in data relaying using some linear coding on the received signals.

\section{A. Signal Model}

The whole system still has two phases. In the first phase, all the users simultaneously broadcast a block of data $\mathbf{s}_{k}=$ $\left(s_{k 1}, s_{k 2}, \cdots, s_{k T}\right)^{T}$ containing $T(>L)$ symbols. Suppose the channel is quasi-static, i.e., the channel coefficients stay constant during a block interval, then the received signal vector at the $l$ th relay and at the destination can be respectively expressed as

$$
\left\{\begin{array}{l}
\mathbf{y}_{s r_{l}}=\sqrt{P \lambda_{s r}} \sum_{k=1}^{K} f_{k l} \mathbf{s}_{k}+\mathbf{n}_{s r_{l}}, \\
\mathbf{y}_{s d}=\sqrt{P \lambda_{s d}} \sum_{k=1}^{K} h_{k} \mathbf{s}_{k}+\mathbf{n}_{s d}
\end{array}\right.
$$

where $\mathbf{n}_{s r_{l}}, \mathbf{n}_{s d} \sim \mathcal{C N}(\mathbf{0}, \mathbf{I})$. Then each relay node performs linear coding on the received signal, and the transmitted signal at the $l$ th relay node is $\mathbf{x}_{r_{l}}=\sqrt{\alpha_{l} P} \mathbf{A}_{l} \mathbf{y}_{s r_{l}}$. To simplify the analysis, we assume the $T \times T$ coding matrices $\left\{\mathbf{A}_{l}\right\}$ are unitary, i.e., $\mathbf{A}_{l} \mathbf{A}_{l}^{H}=\mathbf{I}$. The amplification factor $\alpha_{l}$ at the $l$ th relay node is still given by (2) for VGR and by (3) for FGR, respectively. Then in the second phase, all the relay nodes forward their signals simultaneously to the destination while the source nodes stay silent. The received signal at the destination in the second phase is

$$
\begin{aligned}
\mathbf{y}_{r d} & =\sqrt{\lambda_{r d}} \sum_{l=1}^{L} g_{l} \mathbf{x}_{r_{l}}+\mathbf{n}_{r d} \\
& =P \sqrt{\lambda_{r d} \lambda_{s r}} \sum_{k=1}^{K} \mathbf{M}_{k} \mathbf{v}_{k}+\tilde{\mathbf{n}}_{r d}
\end{aligned}
$$

where $\quad \tilde{\mathbf{n}}_{r d} \sim \mathcal{C N}\left(\mathbf{0},\left(\sum_{l=1}^{L} \alpha_{l} P \lambda_{r d}\left|g_{l}\right|^{2}+1\right) \mathbf{I}\right), \quad \mathbf{v}_{k}=$ $\left(\sqrt{\alpha_{1}} f_{k 1} g_{1}, \sqrt{\alpha_{2}} f_{k 2} g_{2}, \cdots, \sqrt{\alpha_{L}} f_{k L} g_{L}\right)^{T}$ is the equivalent channel vector, and $\mathbf{M}_{k}=\left(\mathbf{A}_{1} \mathbf{s}_{k}, \mathbf{A}_{2} \mathbf{s}_{k}, \cdots, \mathbf{A}_{L} \mathbf{s}_{k}\right)$ is the codeword of the $k$ th user. Clearly, $\mathbf{M}_{k}$ plays the role of the space-time code in the multiple antenna systems. In the following, we assume that $\Delta \mathbf{M}_{k}=\left(\mathbf{A}_{1} \Delta \mathbf{s}_{k}, \mathbf{A}_{2} \Delta \mathbf{s}_{k}, \cdots, \mathbf{A}_{L} \Delta \mathbf{s}_{k}\right)$ always have full rank for any $\Delta \mathbf{s}_{k} \neq \mathbf{0}$, which is the sufficient condition to achieve full diversity for the single-user DSTBCFGR systems [11][12].

\section{B. Performance Analysis}

Using the MLD similar to (5), the PEPs are still given by (6) after redefining $W_{d}=\frac{P \lambda_{s d}}{4}\|\Delta \mathbf{S h}\|^{2}$ and $W_{r}=$ $\frac{P^{2} \lambda_{r d} \lambda_{s r}\left\|\sum_{k=1}^{K} \Delta \mathbf{M}_{k} \mathbf{v}_{k}\right\|^{2}}{4\left(P \lambda_{r d} \sum_{l=1}^{L} \alpha_{l}\left|g_{l}\right|^{2}+1\right)}$. After some manipulations, it is easy to show that

$$
\begin{aligned}
E\left[\exp \left(-W_{d}\right)\right] & =\operatorname{det}^{-1}\left(\mathbf{I}+\frac{1}{4} P \lambda_{s d} \Delta \mathbf{S}^{H} \Delta \mathbf{S}\right) \\
& \stackrel{P}{\approx} \frac{4^{r}}{\lambda_{s d}^{r} \prod_{i=1}^{r} \tau_{i}} P^{-r} \leq \frac{4}{P \lambda_{s d} \tau}
\end{aligned}
$$

where $\Delta \mathbf{S}=\left(\Delta \mathbf{s}_{1}, \Delta \mathbf{s}_{2}, \cdots, \Delta \mathbf{s}_{K}\right)$, and $\tau_{i}$ and $r$ are the $i$ th eigen-value and the rank of the matrix $\Delta \mathbf{S}^{H} \Delta \mathbf{S}$, respectively. Note that the error performance is lower bounded by the worst-case PEP, which occurs when $r=1$ and leads to the last inequality, where $\tau$ is the minimum of the eigen-values of all the matrices $\Delta \mathbf{S}^{H} \Delta \mathbf{S}$ for any $\Delta \mathbf{S} \neq \mathbf{0}$. Next we study the term $E\left[\exp \left(-W_{r}\right)\right]$. For FGR, we have $W_{r, F G R}=$ $\frac{P^{2} \lambda_{r d} \lambda_{s r}\left\|\sum_{k=1}^{K} \Delta \mathbf{M}_{k} \mathbf{D}(\mathbf{g}) \mathbf{f}_{k r}\right\|^{2}}{4\left(P \lambda_{r d} \sum_{l=1}^{L}\left|g_{l}\right|^{2}+K P \lambda_{s r}+1\right)}$, where $\mathbf{f}_{k r}=\left(f_{k 1}, f_{k 2}, \cdots, f_{k L}\right)^{T}$ and $\mathbf{D}(\mathbf{g})=\operatorname{diag}\left(g_{1}, g_{2}, \cdots, g_{L}\right)$. It is easy to show that the expectation of $\exp \left(-W_{r, F G R}\right)$ over $\mathbf{f}_{k r}$ for $k=1,2, \cdots, K$ is

$$
\begin{aligned}
& E_{\left\{\mathbf{f}_{k r}\right\}}\left[\operatorname { e x p } \left(-W_{r, F G R)]}\right.\right. \\
& =\operatorname{det}^{-1}\left(\mathbf{I}+\frac{P^{2} \lambda_{r d} \lambda_{s r} \sum_{k=1}^{K} \Delta \mathbf{M}_{k} \mathbf{D}(\mathbf{g}) \mathbf{D}^{H}(\mathbf{g}) \Delta \mathbf{M}_{k}^{H}}{4\left(P \lambda_{r d} \sum_{l=1}^{L}\left|g_{l}\right|^{2}+K P \lambda_{s r}+1\right)}\right) \\
& \leq \prod_{k=1}^{L}\left(1+\frac{P \lambda_{r d} \lambda_{s r} \eta\left|g_{k}\right|^{2}}{4\left(\lambda_{r d} \sum_{l=1}^{L}\left|g_{l}\right|^{2}+K \lambda_{s r}+1\right)}\right)^{-1}
\end{aligned}
$$

where we use the inequality $\operatorname{det}(\mathbf{A}+\mathbf{B}) \geq \operatorname{det} \mathbf{A}+\operatorname{det} \mathbf{B}$ for any $\mathbf{A}, \mathbf{B} \geq \mathbf{0}$, and $\eta$ is the minimum of the eigen-values of all the matrices $\Delta \mathbf{M}^{H} \Delta \mathbf{M}$ for any $\Delta \mathbf{M} \neq \mathbf{0}$. When there is a large number of relay nodes, we can simplify the above expression by using the approximation $\sum_{k=1}^{L}\left|g_{l}\right|^{2} \stackrel{\gtrsim}{2} L$ without affecting the diversity performances $[11][12]$. Now we can 
obtain

$$
\begin{aligned}
E\left[\exp \left(-W_{r, F G R}\right)\right] & \leq \prod_{k=1}^{L} E_{g_{k}}\left[\left(1+P \delta\left|g_{k}\right|^{2}\right)^{-1}\right] \\
& =\prod_{k=1}^{L}\left[\frac{1}{P \delta} \exp \left(\frac{1}{P \delta}\right) E_{1}\left(\frac{1}{P \delta}\right)\right] \\
& \leq \prod_{k=1}^{L}\left[\frac{1}{P \delta} \log (1+P \delta)\right] \\
P & \stackrel{\approx}{\approx} \frac{1}{\delta^{L}}\left(\frac{\log P}{P}\right)^{L}
\end{aligned}
$$

where $\delta=\frac{\lambda_{r d} \lambda_{s r} \eta}{4\left(L \lambda_{r d}+K \lambda_{s r}+1\right)}$, and we use $e^{x} E_{1}(x) \leq$ $\log \left(1+\frac{1}{x}\right)[24,5.1 .20]$ in the last inequality. Plugging (22) and (24) back into (6), we have

$$
\operatorname{Pr}(\mathbf{s} \rightarrow \hat{\mathbf{s}} \mid \operatorname{DSTBC}-\mathrm{FGR}) \stackrel{P \rightarrow \infty}{\approx} \frac{4}{\delta^{L} \lambda_{s d} \tau} \frac{(\log P)^{L}}{P^{L+1}} .
$$

It is observed that the diversity gain of DSTBC-FGR is $(L+1,-L)$ and it is independent of the number of users; however, there is some loss of coding gain compared to the single-user case as $\delta$ is a decreasing function of $K$.

Next we study DSTBC-VGR. Consider a specific error pair where $\Delta \mathbf{s}_{k}=\mathbf{0}$ and $\Delta \mathbf{M}_{k}=\mathbf{0}$ for $k=2,3, \cdots, K$. Then we have $W_{d}=\frac{P \lambda_{s d}\left\|\Delta \mathbf{s}_{1}\right\|^{2}}{4}\left|h_{1}\right|^{2}$. Let $\rho$ be the maximum eigenvalue of $\Delta \mathbf{M}_{1}^{H} \Delta \mathbf{M}_{1}$, and without loss of generality, suppose $\left|g_{1}\right|^{2} \geq\left|g_{2}\right|^{2} \geq \cdots \geq\left|g_{L}\right|^{2}$, then we can obtain

$$
\begin{aligned}
W_{r, V G R} & =\frac{P^{2} \lambda_{r d} \lambda_{s r}\left\|\Delta \mathbf{M} \mathbf{v}_{1}\right\|^{2}}{4\left(P \lambda_{r d} \sum_{l=1}^{L} \alpha_{l, V G R}\left|g_{l}\right|^{2}+1\right)} \\
& \leq \frac{\rho L P \lambda_{r d}\left|g_{1}\right|^{2}\left(P \lambda_{s r} \sum_{k=1}^{K}\left|f_{k 1}\right|^{2}+1\right)}{4\left(P \lambda_{r d}\left|g_{1}\right|^{2}+P \lambda_{s r} \sum_{k=1}^{K}\left|f_{k 1}\right|^{2}+1\right)} \\
\leq & \frac{\rho L}{4} \min \left(P \lambda_{r d}\left|g_{1}\right|^{2}, P \lambda_{s r} \sum_{k=1}^{K}\left|f_{k 1}\right|^{2}+1\right)
\end{aligned}
$$

where we use $P \lambda_{s r} \alpha_{l, V G R}\left|f_{1 l}\right|^{2} \leq 1, \sum_{l=1}^{L}\left|g_{l}\right|^{2} \leq L\left|g_{1}\right|^{2}$ and $\sum_{l=1}^{L} \alpha_{l, V G R}\left|g_{l}\right|^{2} \geq \alpha_{1, V G R}\left|g_{1}\right|^{2}$ in $(a)$. Now we can lower bound the PEP by $\operatorname{Pr}(\mathbf{s} \rightarrow \hat{\mathbf{s}} \mid \mathrm{DSTBC}-\mathrm{VGR}) \geq \max \left(F_{1}, F_{2}\right)$, where

$$
\begin{aligned}
& F_{1}=E\left[Q\left(\sqrt{\mu P\left(\left|h_{1}\right|^{2}+\left|g_{1}\right|^{2}\right)}\right)\right] \\
& P \underset{\approx}{\approx} \frac{(2 L+1) !}{(L+1) !}\left(\frac{8}{\mu}\right)^{L+1} P^{-(L+1)} \\
& F_{2}=E\left[Q\left(\sqrt{\mu\left(P\left|h_{1}\right|^{2}+P \sum_{k=1}^{K}\left|f_{k 1}\right|^{2}+1\right)}\right)\right] \\
& \stackrel{P}{\underset{\approx}{*}} C\left(\frac{2}{\mu}\right)^{K+1} P^{-(K+1)}
\end{aligned}
$$

where $\mu=\frac{1}{4} \max \left(\lambda_{s d}\left\|\Delta \mathbf{s}_{1}\right\|^{2}, \rho L \lambda_{r d}, \rho L \lambda_{s r}, \rho L\right)$, and $C=$ $\frac{1}{\pi} \int_{0}^{\frac{\pi}{2}} \exp \left(-\frac{\mu}{2 \sin ^{2} \theta}\right)(\sin \theta)^{2(K+1)} d \theta$ is some constant. Consequently, we have

$$
\operatorname{Pr}(\mathbf{s} \rightarrow \hat{\mathbf{s}} \mid \text { DSTBC-VGR }) \geq \mathrm{O}\left(P^{-\min (K+1, L+1)}\right)
$$

i.e., the diversity gain of DSTBC-VGR is upper bounded by $\min (K+1, L+1)$.

Comparing (25) and (29), we can observe that the diversity performances of DSTBC-FGR and DSTBC-VGR are very distinct. To be specific, full diversity is always achieved by DSTBC-FGR, whereas the diversity of DSTBC-VGR is also upper bounded by the number of users. This phenomenon is caused by the noise enhancement effect of VGR, as can be seen by studying the noise power (denoted by $\sigma^{2}$ ) of the relay-destination signal (21). For DSTBC-FGR, we have $\sigma_{F G R}^{2}=\sum_{l=1}^{L} \frac{P \lambda_{r d}\left|g_{l}\right|^{2}}{K P \lambda_{s r}+1}+1 \stackrel{P}{{ }^{2}}{ }^{\infty} \sum_{l=1}^{L} \frac{\lambda_{r d}\left|g_{l}\right|^{2}}{K \lambda_{s r}}+1$, which is independent of the transmitted power $P$ and the userrelay channels. On the contrary, for DSTBC-VGR we have $\sigma_{V G R}^{2}=\sum_{l=1}^{L} \frac{P \lambda_{r d}\left|g_{l}\right|^{2}}{P \lambda_{s r} \sum_{k=1}^{K}\left|f_{k l}\right|^{2}+1}+1$. As a result, the noise power would be comparable to the transmitted power $P$ whenever $P \lambda_{s r} \sum_{k=1}^{K}\left|f_{k l}\right|^{2}=\mathrm{O}(1)$, in which case the decoding error is likely to occur at the receiver with very high probability. It is easy to show that the probability of such dominant error events is $\mathrm{O}\left(P^{-K}\right)$, thus the overall diversity of DSTBC-VGR is also upper bounded by $(K+1)$.

\section{Selective DSTBC-VGR for Single-User Networks}

So far, we have shown that the diversity of DSTBC-VGR is upper bounded by the number of users. For the conventional single-user analog relaying networks (i.e., $K=1$ ), this implies that the diversity order is limited by 2 regardless of the number of relay nodes. We have also seen that the noise enhancement effect is the main cause of the diversity loss, which happens when all the channels from the users to a certain relay node experience deep fading. Note that we have implicitly assumed that the relay nodes are using full power all the time. Intuitively, if the input channels are in bad conditions, the relay nodes should lower its transmitted power or even stay idle to mitigate the noise enhancement effect. In the following, we shall adopt this idea and develop the selective DSTBCVGR to recover the diversity loss when there is only one user (i.e., $K=1$ ).

Since the quality of the relay branch is uniquely characterized by $W_{r, \mathrm{VGR}}$, we first rewrite this term as $W_{r, V G R}=$ $\frac{\kappa P \lambda_{s r} \sum_{l=1}^{L} a_{l}\left|f_{1 l}\right|^{2} \nu_{l}}{4\left(\sum_{l=1}^{L} a_{l} \nu_{l}+1\right)}$, where $\kappa \in[\eta, \rho]$ is a constant, $\nu_{l}=$ $\frac{P \lambda_{r d}\left|g_{l}\right|^{2}}{P \lambda_{s r}\left|f_{1 l}\right|^{2}+1}$ and $a_{l} \leq 1$ is the power scaling coefficient. Clearly, the best power allocation scheme is to maximize 


$$
\begin{aligned}
& W_{r, V G R} \text {, i.e., } \\
& \max g(\mathbf{a}) \triangleq \frac{\sum_{l=1}^{L} a_{l}\left|f_{1 l}\right|^{2} \nu_{l}}{\sum_{l=1}^{L} a_{l} \nu_{l}+1}, \text { s.t. } a_{l} \leq 1 \text { for } l=1,2, \cdots, L,
\end{aligned}
$$

where $\mathbf{a}=\left(a_{1}, a_{2}, \cdots, a_{L}\right)^{T}$. Although the above optimization problem is also studied in [9], the authors only propose an iterative algorithm to search for the optimizer and no performance analysis is performed there. Unlike that work, we give the closed-form solution to the above problem and prove that full diversity can be achieved. Without loss of generality, we assume $\left|f_{11}\right|^{2}>\left|f_{12}\right|^{2}>\cdots>\left|f_{1 L}\right|^{2}$ and define $f_{1(L+1)}=0$.

Proposition 2: The solution to the problem (30) is

$$
a_{l}^{*}=\left\{\begin{array}{ll}
1, l=1,2, \cdots, l_{0} \\
0, & \text { o.w. }
\end{array},\right.
$$

where $l_{0} \in\{1,2, \cdots, L\}$ is the smallest index satisfying $\sum_{l=1}^{l_{0}}\left(\left|f_{1 l}\right|^{2}-\left|f_{1\left(l_{0}+1\right)}\right|^{2}\right) \nu_{l} \geq\left|f_{1\left(l_{0}+1\right)}\right|^{2}$. Besides, the diversity gain of such selective DSTBC-VGR is $(L+1,0)$.

Proof: In [9], it has been proved that $a_{l}^{*} \in\{0,1\}$. We now prove by contradiction that if $a_{l_{1}}^{*}=1$, then $a_{l_{2}}^{*}=1$ for any $l_{2} \leq l_{1}$. Suppose now there exists an $a_{l_{2}}^{*}=0$ with $l_{2}<l_{1}$. Then we can always find a sufficiently small quantity $\delta \ll 1$ such that $\frac{\delta \nu_{l_{1}}}{\nu_{l_{2}}} \leq 1$. Consequently, there exists another feasible solution $\hat{\mathbf{a}}$ with $\hat{a}_{l_{1}}=a_{l_{1}}^{*}-\delta$ and $\hat{a}_{l_{2}}=\frac{\delta \nu_{l_{1}}}{\nu l_{2}}$, and the other elements of $\hat{\mathbf{a}}$ are the same as $\mathbf{a}^{*}$. It is easy to check that $g(\hat{\mathbf{a}})-g\left(\mathbf{a}^{*}\right)=\frac{\delta \nu_{l_{1}}\left(\left|f_{1 l_{2}}\right|^{2}-\left|f_{1 l_{1}}\right|^{2}\right)}{\sum_{l=1}^{L} a_{l}^{*} \nu_{l}+1}>0$, which contradicts the optimality of $\mathbf{a}^{*}$. Now the optimizer can be limited to the finite set $\left\{\mathbf{a}_{1}, \mathbf{a}_{2}, \cdots, \mathbf{a}_{L}\right\}$, where $\mathbf{a}_{l}=\left(\mathbf{1}_{l \times 1}, \mathbf{0}_{(L-l) \times 1}\right)$. After some manipulations, we can show that $g\left(\mathbf{a}_{k}\right) \geq g\left(\mathbf{a}_{k+1}\right)$ is equivalent to the condition $\sum_{l=1}^{k}\left(\left|f_{1 l}\right|^{2}-\left|f_{1(k+1)}\right|^{2}\right) \nu_{l} \geq\left|f_{1(k+1)}\right|^{2}$, where the left-hand side is increasing with $k$ and the right-hand side is decreasing with $k$. Consequently, we have $g\left(\mathbf{a}_{1}\right) \leq g\left(\mathbf{a}_{2}\right) \leq \cdots \leq$ $g\left(\mathbf{a}_{l_{0}}\right)$ and $g\left(\mathbf{a}_{l_{0}}\right) \geq g\left(\mathbf{a}_{l_{0}+1}\right) \geq \cdots \geq g\left(\mathbf{a}_{L+1}\right)$, which completes the proof of the first part. To show the diversity performance, we note that the vectors $\left\{\mathbf{e}_{1}, \mathbf{e}_{2}, \cdots, \mathbf{e}_{L}\right\}$ also belong to the feasible set, where $\mathbf{e}_{l}$ is a $L \times 1$ vector with the $k$ th element being 1 and the other elements being 0 . Note that each $\mathbf{e}_{l}$ corresponds to the case when only the $l$ th relay is selected to forward data. Consequently, the selective DSTBCVGR performs strictly better than the best relay selection scheme, which achieves the diversity gain $(L+1,0)$ [10].

Basically, the above results show that each relay node either stays idle or forwards data with full power, and the relays with better user-relay channel conditions have the priority to be selected. These facts indicate an easy implementation of the proposed selective DSTBC strategy, i.e., the destination can first compute the active relay set and then feed back a single threshold. The relays whose input channel conditions are better than the threshold then stay active in the second phase. On the contrary, for the iterative algorithm proposed in [9], the destination has to feed back the whole active relay set, and the feedback overhead is formidable when there is a large number of relays.

\section{Diagonal Distributed Space-Time Coding}

In this section, we study DDSTC that can achieve full diversity for both VGR and FGR. Different from DSTBC, where the unitary coding matrices are employed at the relay nodes, DDSTC has a diagonal structure by letting only one relay forward data at each time instant.

The whole data transmission still takes place in two phases. In the first phase, all the users broadcast a set of $L$ symbols. The received signals at the $l$ th relay node and at the destination are given by (20a) and by (20b), respectively. Then each relay node performs linear transformation on the received signal vector, and the transmitted signal at the $l$ th relay node is $x_{r_{l}}=$ $\sqrt{\alpha_{l} L P} \mathbf{t}_{l}^{T} \mathbf{y}_{s r_{l}}$. Here $\mathbf{t}_{l}$ is the $L \times 1$ coding vector having unit norm (i.e., $\left\|\mathbf{t}_{l}\right\|^{2}=1$ ), the design criterion of which will be clear later. The constant $L$ is introduced to normalize the total transmitted power, as each relay node only forwards data in one time slot in the second phase. The amplification factor $\alpha_{l}$ at the $l$ th relay node is still given by (2) for VGR and by (3) for FGR, respectively. Then in the second phase, all the relay nodes take turns to forward data, and the received signal at the destination during the $l$ th time slot is

$$
\begin{aligned}
y_{r_{l} d} & =\sqrt{\lambda_{r d}} g_{l} x_{r_{l}}+n_{r_{l} d} \\
& =P \sqrt{L \lambda_{s r} \lambda_{r d} \alpha_{l}} g_{l} \sum_{k=1}^{K} f_{k l} \mathbf{t}_{l}^{T} \mathbf{s}_{k}+\tilde{n}_{r_{l} d},
\end{aligned}
$$

where $\tilde{n}_{r_{l} d} \sim \mathcal{C N}\left(0, L P \lambda_{r d} \alpha_{l}\left|g_{l}\right|^{2}+1\right)$ is the equivalent additive noise. Upon observing the signals $\mathbf{y}_{s d}$ and $\left\{y_{r_{l} d}\right\}$, the destination performs MLD to jointly decode the $K$ user symbols as

$$
\begin{aligned}
\mathbf{s}_{d}= & \arg \min _{\hat{\mathbf{s}}_{k} \in \Omega^{L}}\left\|\mathbf{y}_{s d}-\sqrt{P \lambda_{s d}} \sum_{k=1}^{K} h_{k} \hat{\mathbf{s}}_{k}\right\|^{2} \\
& +\sum_{l=1}^{L} \frac{\left\|y_{r_{l} d}-P \sqrt{L \lambda_{s r} \lambda_{r d} \alpha_{l}} g_{l} \sum_{k=1}^{K} f_{k l} \mathbf{t}_{l}^{T} \hat{\mathbf{s}}_{k}\right\|^{2}}{L P \lambda_{r d} \alpha_{l}\left|g_{l}\right|^{2}+1},
\end{aligned}
$$

and the PEP is given by

$$
\begin{aligned}
\operatorname{Pr}(\mathbf{s} \rightarrow \hat{\mathbf{s}}) & =E\left[Q\left(\sqrt{2\left(W_{d}+\sum_{l=1}^{L} W_{r_{l}}\right)}\right)\right] \\
& \leq E\left[\exp \left(-W_{d}-\sum_{l=1}^{L} W_{r_{l}}\right)\right]
\end{aligned}
$$

where $\quad W_{d}=\frac{P \lambda_{s d}}{4}\|\Delta \mathbf{S h}\|^{2}, \quad W_{r_{l}}=$ $\frac{\alpha_{l} P^{2} L \lambda_{s r} \lambda_{r d}\left|g_{l}\right|^{2}\left|\mathbf{f}_{s l}^{T} \Delta \mathbf{S}^{T} \mathbf{t}_{l}\right|^{2}}{4\left(L P \lambda_{r d} \alpha_{l}\left|g_{l}\right|^{2}+1\right)}, \quad \mathbf{f}_{s l}=\left(f_{1 l}, f_{2 l}, \cdots, f_{K l}\right)^{T}$ and $\Delta \mathbf{S}=\left(\Delta \mathbf{s}_{1}, \Delta \mathbf{s}_{2}, \cdots, \Delta \mathbf{s}_{K}\right)$. The first term $E\left[\exp \left(-W_{d}\right)\right]$ has been given by (22). As for the second term, we observe that each $W_{r_{l}}$ has the similar form as $W_{r}$ in the single-relay case discussed in Section II-B. By following the same steps, we can obtain

$$
E\left[\exp \left(-W_{r_{l}}\right)\right] \stackrel{P \rightarrow \infty}{\leq} \frac{4 K}{L \lambda_{r d}\left\|\Delta \mathbf{S}^{T} \mathbf{t}_{l}\right\|^{2}} \frac{\log P}{P}
$$


for both the VGR and FGR. Plugging (22) and (35) back into (34) leads to

$$
\operatorname{Pr}(\mathbf{s} \rightarrow \hat{\mathbf{s}}) \stackrel{P \rightarrow \infty}{\leq} \frac{4^{L+1}}{\lambda_{s d} \tau}\left(\frac{K}{L \lambda_{r d}}\right)^{L}\left(\prod_{l=1}^{L}\left|\Delta \mathbf{s}_{k}^{T} \mathbf{t}_{l}\right|^{2}\right)^{-1} \frac{(\log P)^{L}}{P^{L+1}}
$$

where we use $\prod_{l=1}^{L}\left\|\Delta \mathbf{S}^{T} \mathbf{t}_{l}\right\|^{2} \geq \prod_{l=1}^{L}\left|\Delta \mathbf{s}_{k}^{T} \mathbf{t}_{l}\right|^{2}$ in the inequality, i.e., the dominant error events occur when only one user symbol vector is decoded incorrectly. Unlike DSTBC, we observe that both of DDSTC-VGR and DDSTC-FGR can achieve the diversity gain $(L+1,-L)$. This is because the diagonal structure of DDSTC can efficiently mitigate the noise enhancement effect, as the output noises of the relay nodes would not be combined concurrently at the destination. Besides, we conclude that the best code design criterion is to maximize the minimum product distance, i.e.,

$$
\max _{\mathbf{t}_{l} \in \mathbb{C}^{L \times 1},\left\|\mathbf{t}_{l}\right\|^{2}=1} \min _{\Delta \mathbf{s} \neq \mathbf{0}} \prod_{l=1}^{L}\left|\Delta \mathbf{s}^{T} \mathbf{t}_{l}\right|^{2},
$$

which has been well studied in the literatures. For example, when $L=2^{s}(s \geq 1)$ and the signal constellation $\Omega$ has the form $\mathbb{Z}[j]=\{a+j b \mid a, b \in \mathbb{Z}, j=\sqrt{-1}\}$, the optimum coding matrix is given by [13] $\mathbf{T}_{\mathrm{opt}}=\frac{1}{\sqrt{L}} \mathbf{V}\left(\theta_{1}, \theta_{2}, \cdots, \theta_{L}\right)$, where $\mathbf{T}=\left(\mathbf{t}_{1}, \mathbf{t}_{2}, \cdots, \mathbf{t}_{L}\right)^{T}, \theta_{l}=e^{j \frac{4 l-3}{2 L} \pi}$ for $l=1,2, \cdots, L$, and

$$
\mathbf{V}\left(\theta_{1}, \theta_{2}, \ldots, \theta_{L}\right)=\left(\begin{array}{cccc}
1 & \theta_{1} & \cdots & \theta_{1}^{L-1} \\
1 & \theta_{2} & \cdots & \theta_{2}^{L-1} \\
\vdots & \vdots & \ddots & \vdots \\
1 & \theta_{L} & \cdots & \theta_{L}^{L-1}
\end{array}\right)
$$

is the $L \times L$ Vandermonde matrix with parameters $\theta_{1}, \theta_{2}, \cdots, \theta_{L}$. For more code designs, please refer to [14] and the references therein.

\section{Simulations}

In this section, we shall present some simulation results to validate our analysis. We use the path loss model $\lambda=D^{-3}$, where $\lambda$ is the path-loss coefficient and $D$ is the distance between two terminals. Pair error probability is used as the performance metric, i.e., the probability that at least one of the user symbols is decoded incorrectly at the destination. To simplify the simulation settings, only symmetric networks with one or two users are considered, and $D_{s d}$ is always normalized to 1 .

Fig. 2 shows the error performances with different channel conditions, where the two users use QPSK signals and there is only one relay node. Compared with direct transmission (DT), a diversity order of 2 is achieved due to node cooperation. We observe that VGR has about 1dB SNR gain over FGR in all cases. It is also observed that the error performances almost remain unchanged after improving the qualities of user-relay channels, whereas about $3 \mathrm{~dB}$ SNR gain is achieved when the relay-destination channels become better, which is consistent with our analysis that the relay-destination channel conditions dominate the error performances.

In Fig. 3, we compare with the conventional single-user (i.e., $K=1$ ) analog relaying [2], where all the users are

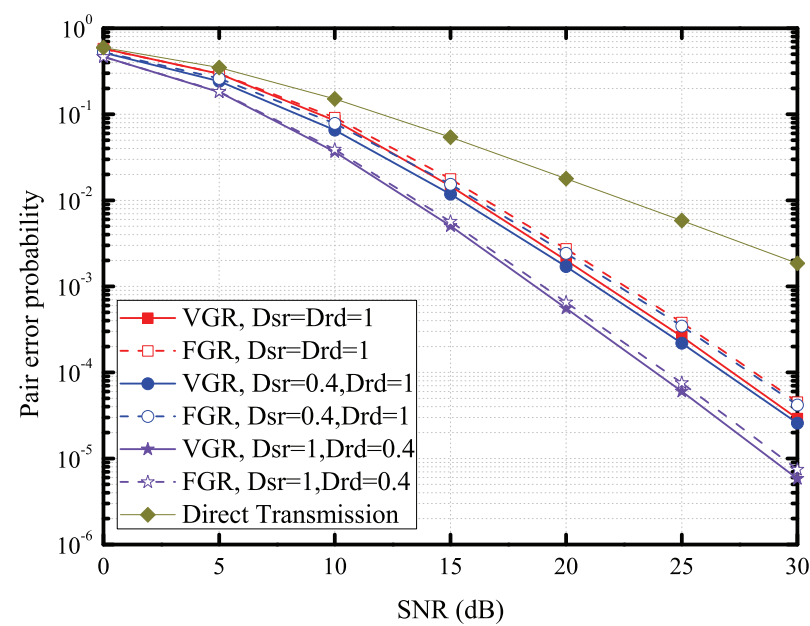

Fig. 2. Error performances of a two-user network with different channel conditions.

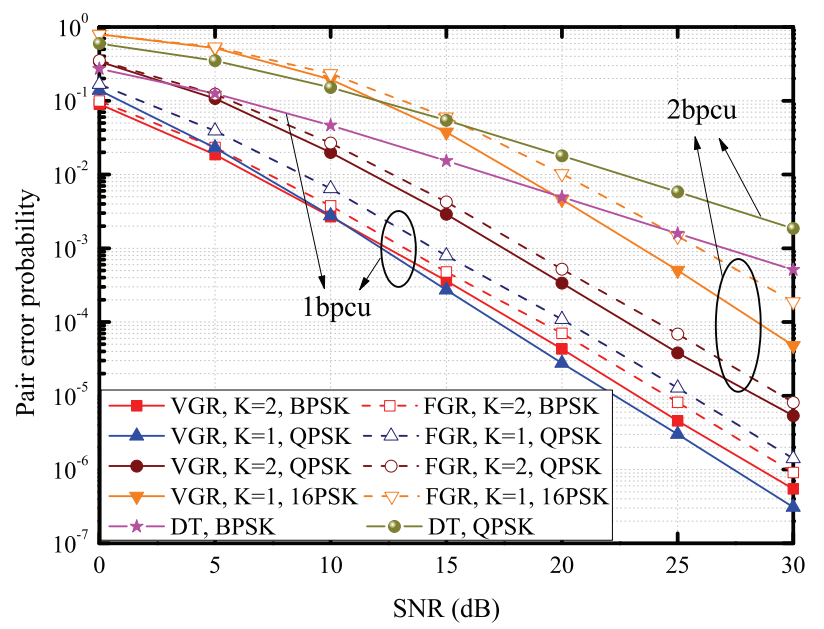

Fig. 3. Comparison of two-user and single-user network with different data rate.

served separately in a time-sharing manner. The transmitted power and the total data rate have been properly normalized, and for network topology we let $D_{s r}=D_{r d}=0.5$. We observe that full diversity is achieved in all cases. However, the single-user VGR is superior to all the other schemes when the total data rate is only 1 bit per channel use (bpcu) because there is no logarithmic-term loss at modest SNRs. When the data rate is $2 \mathrm{bpcu}$, although the error rate of single-user VGR still decreases faster, the spectral efficiency dominates the overall performances and thus the two-user systems show huge performance gain.

Next we study the error performances of relay selection in Fig. 4, where QPSK signal is employed. As the reference, we also simulate Ding's scheme [20] for VGR, where

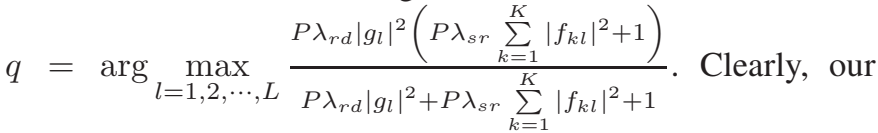
Min-Max scheme can achieve full diversity for both VGR and FGR, whereas the diversity of Ding's scheme is bounded by 2 . This is because in Ding's scheme, the channel phases have nothing to do with relay selection. However, the channel phases actually have tremendous effects on PEPs, since the 


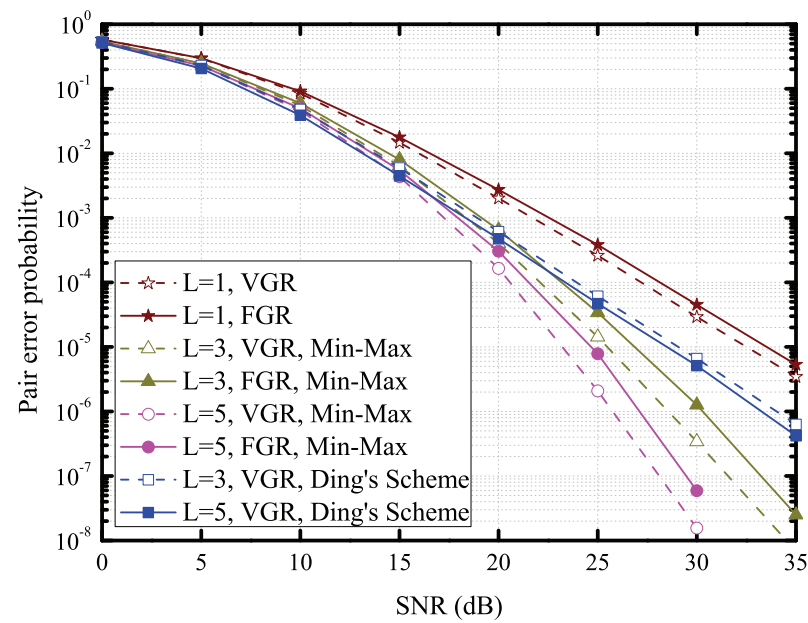

Fig. 4. Error performances of a two-user network with relay selection.

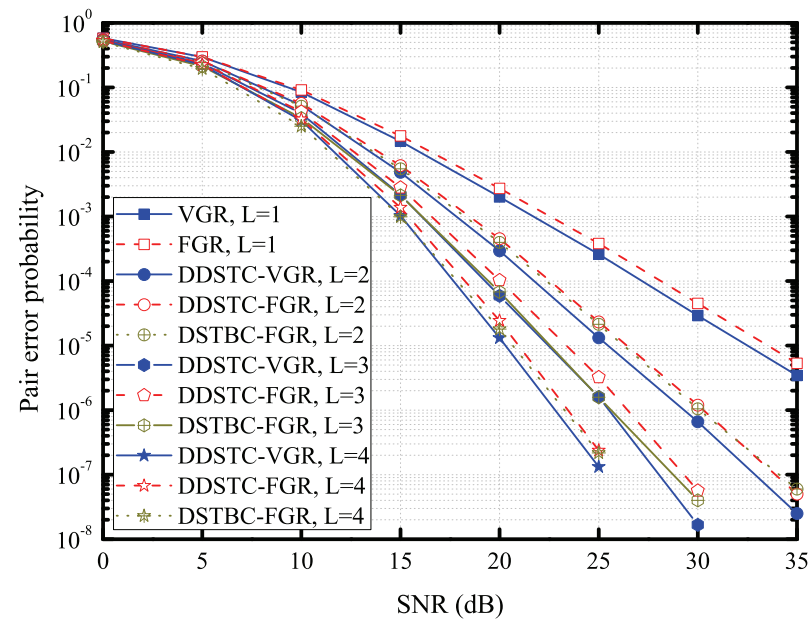

Fig. 5. Error performances of a two-user network with DDSTC and DSTBCFGR.

source messages are randomly mixed in the air and the MUI depends largely on the orthogonality of the instant channel coefficients. Although Ding's scheme does achieve full diversity in terms of outage capacity [20], where successive interference cancelation is employed at the destination and error-free decoding is assumed, our Min-Max strategy is more practical for real systems where coding/decoding is no longer ideal.

In Fig. 5, we show the performances of DSTBC-FGR and DDSTC with two users, where QPSK signal is employed. We observe that both coding schemes can achieve full diversity with multiple relays. Comparatively, DSTBC-FGR performs slightly better than DDSTC-FGR, since the diagonal structure of DDSTC limits the minimum distances of the codeword. About 1dB SNR gain is achieved by using DDSTC-VGR against DDSTC-FGR regardless of the number of relays. Comparing Fig. 4 and Fig. 5, we observe that DSTC can achieve much higher coding gain than relay selection does, as all the relay nodes are contributing to forward data.

Finally we study the performances of DSTBC-VGR using QPSK signal. When there are two users, we observe in Fig. 6 that the performance of DSTBC-VGR is bounded by that of

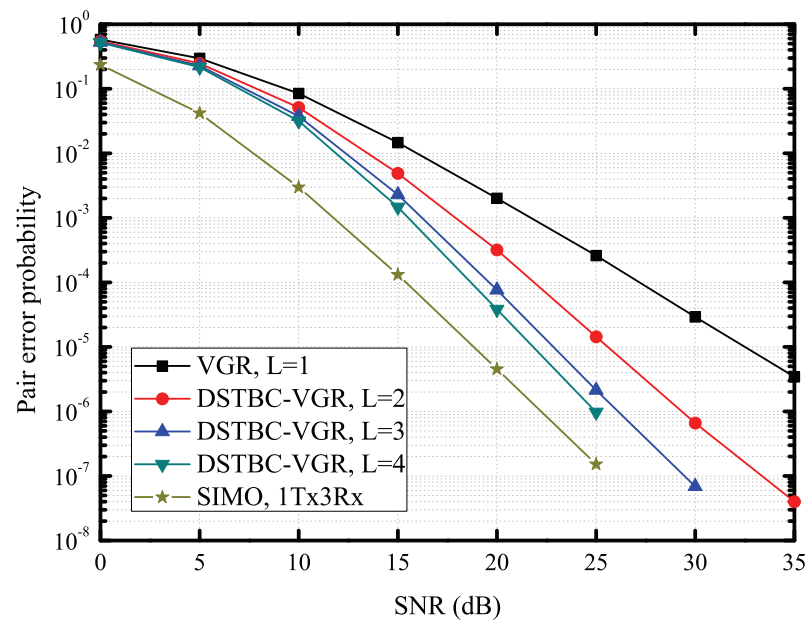

Fig. 6. Error performances of a two-user network with DSTBC-VGR.

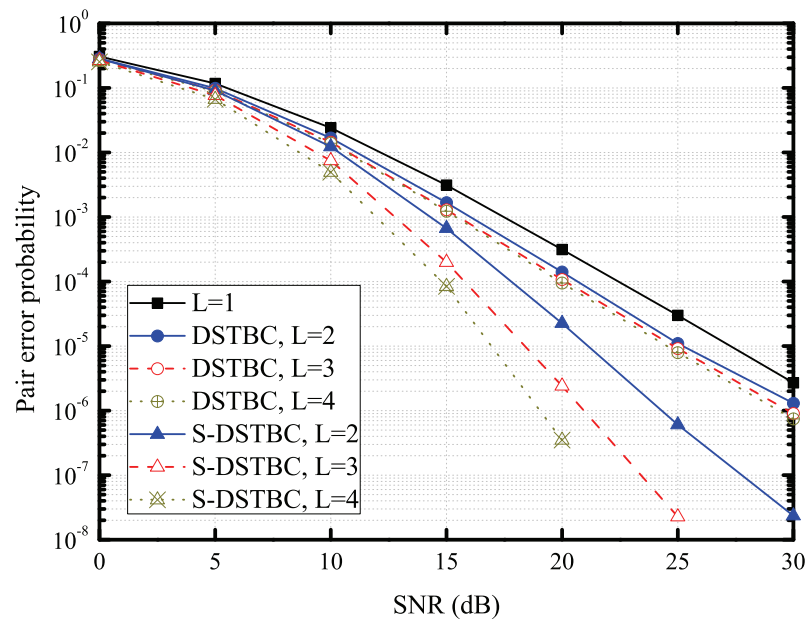

Fig. 7. Error performances of a single-user network with DSTBC-VGR and selective DSTBC-VGR.

the single-input multiple-output systems with one transmitted antenna and three received antennas (1Tx3Rx), which is well known to have a diversity order of 3 . As for the single-user systems shown in Fig. 7, it is observed that the diversity order is always 2, and the marginal coding gain is very trivial by increasing the number of relay nodes beyond 2. Clearly, our selective DSTBC (S-DSTBC) can fully recover the diversity loss with very small signalling overhead.

\section{CONCLUSIONS AND Future WORK}

In this work, we have studied the diversity performances of the multi-user network-coded systems using analog relaying transmission. We showed that full diversity can be achieved through relay selection and DSTC, but the MUI at the intended receiver will induce the logarithmic-term loss and degrade the diversity performances at modest SNRs. For future work, one may study the code design for DSTBC and extend our selective DSTBC scheme to the multi-user networks. One may also investigate other distributed beamforming schemes to improve the coding gain. 


$$
\operatorname{Pr}(w \leq T(w) \leq c w Z+w) \leq \frac{2 c w}{a b} \exp \left(-\frac{a+b}{a b} w\right) \sum_{k=0}^{N-1} \frac{1}{k !} \int_{0}^{\infty} t^{k} e^{-t} K_{0}\left(2 \sqrt{\frac{c w t}{a b}}\right) d t
$$

\section{APPENDIX}

\section{A. Proof of Lemma 1}

For $W_{2}$, it is easy to show that

$$
\begin{aligned}
F_{W_{2}}(w)=\operatorname{Pr} & \left(X \leq \frac{w c}{(b Y-w) a}, b Y-w>0\right) \\
& +\operatorname{Pr}(b Y-w<0) .
\end{aligned}
$$

The first term is given by $\operatorname{Pr}(b Y-w<0)=1-\exp \left(-\frac{w}{b}\right)$. For the second term, we have

$$
\begin{aligned}
& \operatorname{Pr}\left(X \leq \frac{w c}{(b Y-w) a}, b Y-w>0\right) \\
& \quad=\exp \left(-\frac{w}{b}\right)-\exp \left(-\frac{w}{b}\right) \sqrt{\frac{4 w c}{a b}} K_{1}\left(\sqrt{\frac{4 w c}{a b}}\right)
\end{aligned}
$$

where $K_{1}(x)$ is the first-order modified Bessel function of the second kind [24, 9.6.1], and we use [25, 3.478.4] in the equality. Combining these two terms leads to the first part of (9), which is consistent with a previous result derived using different algebra [27]. Next we examine the asymptotic behaviors of $F_{W_{2}}(w)$ when $w \ll 1$. Using [24, 9.6.11], we have $K_{1}(z) \stackrel{z \ll 1}{\approx} z^{-1}+\log \left(\frac{1}{2} z\right) I_{1}(z)$, where $I_{1}(z)$ is the firstorder modified Bessel function of the first kind [24, 9.6.1], which can be further approximated as $I_{1}(z) \stackrel{z \ll 1}{\approx} \frac{z}{2}[24,9.6 .7]$. Therefore we have

$$
z K_{1}(z) \stackrel{z \ll 1}{\approx} 1+\frac{1}{2} z^{2} \log z .
$$

Using the above approximation, we can obtain

$$
\begin{aligned}
F_{W_{2}}(w) \stackrel{w}{ } \stackrel{\geqq 1}{\approx} 1-\left(1-\frac{w}{b}\right)\left(1+\frac{2 w c}{a b} \log \left(\sqrt{\frac{4 w c}{a b}}\right)\right) \\
w \ll 1 \frac{c}{a b} w \log \frac{1}{w} .
\end{aligned}
$$

Next we study $F_{W_{1}}(w)$. Let $T(w)=a b X Y-a w X-b w Y$, then

$$
F_{W_{1}}(w)=\operatorname{Pr}(T(w) \leq w)+\operatorname{Pr}(w \leq T(w) \leq c w Z+w) .
$$

For any $t \geq 0$, we have

$$
\begin{gathered}
F_{T}(t)=\operatorname{Pr}\left(Y \leq \frac{t+a w X}{(a X-w) b}, a X-w \geq 0\right) \\
+\operatorname{Pr}(a X-w \leq 0) .
\end{gathered}
$$

The first term is given by $\operatorname{Pr}(a X-w \leq 0)=1-\exp \left(-\frac{w}{a}\right)$. For the second term, we have

$$
\begin{gathered}
\operatorname{Pr}\left(Y \leq \frac{t+a w X}{(a X-w) b}, a X-w \geq 0\right)=\exp \left(-\frac{w}{a}\right) \\
\quad-\exp \left(-\frac{a+b}{a b} w\right) 2 \sqrt{\frac{t+w^{2}}{a b}} K_{1}\left(2 \sqrt{\frac{t+w^{2}}{a b}}\right)
\end{gathered}
$$

where we use $[25,3.478 .4]$ again. Consequently, for $t \geq 0$

$$
F_{T}(t)=1-\exp \left(-\frac{a+b}{a b} w\right) 2 \sqrt{\frac{t+w^{2}}{a b}} K_{1}\left(2 \sqrt{\frac{t+w^{2}}{a b}}\right) .
$$

Using the relation $K_{\nu}^{\prime}(x)=-K_{\nu-1}(x)-\frac{\nu}{x} K_{\nu}(x)$ [24, 9.6.26], we have

$$
\begin{aligned}
\left(x K_{1}(x)\right)^{\prime} & =K_{1}(x)+x K_{1}^{\prime}(x) \\
& =K_{1}(x)+x\left(-K_{0}(x)-\frac{1}{x} K_{1}(x)\right) \\
& =-x K_{0}(x),
\end{aligned}
$$

where $K_{0}(x)$ is the zeroth-order modified Bessel function of the second kind [24, 9.6.1]. Thus for $t \geq 0$

$$
f_{T}(t)=\frac{2}{a b} \exp \left(-\frac{a+b}{a b} w\right) K_{0}\left(2 \sqrt{\frac{t+w^{2}}{a b}}\right) .
$$

By using the relation

$$
\begin{aligned}
1-F_{Z}(z) & =\frac{1}{\Gamma(N)} \int_{z}^{\infty} t^{N-1} e^{-t} d t \\
& =\frac{\Gamma(N, z)}{\Gamma(N)}=e^{-z} \sum_{k=0}^{N-1} \frac{z^{k}}{k !},
\end{aligned}
$$

where $\Gamma(s, x)$ is the upper incomplete gamma function [24, 6.5.3], we can obtain (50) shown on the top of this page, where we use the fact that $K_{0}(x)$ is a decreasing function in the inequality. To evaluate the integral within the summation, we use [25, 6.643.3] and obtain

$$
\begin{aligned}
\int_{0}^{\infty} & t^{k} e^{-t} K_{0}\left(2 \sqrt{\frac{c w t}{a b}}\right) d t \\
& =\frac{\Gamma^{2}(k+1)}{2 \sqrt{\frac{c w}{a b}}} \exp \left(\frac{c w}{2 a b}\right) W_{-\left(k+\frac{1}{2}\right), 0}\left(\frac{c w}{a b}\right) .
\end{aligned}
$$

Here $W_{\kappa, \mu}(z)=e^{-\frac{1}{2} z} z^{\frac{1}{2}+\mu} U\left(\frac{1}{2}+\mu-\kappa, 1+2 \mu, z\right)$ is the Whittaker's function [24, 13.1.33], and $U(a, b, z)=$ $\frac{1}{\Gamma(a)} \int_{0}^{\infty} e^{-z t} t^{a-1}(1+t)^{b-a-1} d t$ is the Kummer's function $[24,13.2 .5]$. Now we have

$$
\begin{aligned}
\int_{0}^{\infty} & t^{k} e^{-t} K_{0}\left(2 \sqrt{\frac{c w t}{a b}}\right) d t \\
& =\frac{1}{2} \Gamma(k+1) \int_{0}^{\infty} e^{-\frac{c w}{a b} t} \frac{t^{k}}{(1+t)^{k+1}} d t \\
& \leq \frac{1}{2} \Gamma(k+1) \int_{0}^{\infty} \frac{\exp \left(-\frac{c w}{a b} t\right)}{1+t} d t \\
& =\frac{1}{2} \Gamma(k+1) \exp \left(\frac{c w}{a b}\right) E_{1}\left(\frac{c w}{a b}\right),
\end{aligned}
$$

where $E_{1}(x)=\int_{x}^{\infty} \frac{e^{-t}}{t} d t$ is the exponential integral function $[24,5.1 .1]$. Finally we have

$$
\begin{aligned}
\operatorname{Pr} & (w \leq T(w) \leq c w Z+w) \\
& \leq \frac{N c w}{a b} \exp \left(-\frac{a+b-c}{a b} w\right) E_{1}\left(\frac{c w}{a b}\right) .
\end{aligned}
$$


Plugging (46) and (53) back into (43) leads to the first part of (8). Using (41), we have

$$
\begin{aligned}
F_{T}(w) \stackrel{w}{ } & \stackrel{\ll}{\approx} 1-\left(1-\frac{a+b}{a b} w\right)\left(1+\frac{w}{a b} \log \left(\frac{4 w}{a b}\right)\right) \\
w & \ll 1 \\
& \approx \frac{1}{a b} w \log \frac{1}{w} .
\end{aligned}
$$

Using the inequality $e^{x} E_{1}(x) \leq \log \left(1+\frac{1}{x}\right)[24,5.1 .20]$, we have

$$
\begin{aligned}
\operatorname{Pr} & (w \leq T(w) \leq c w Z+w) \\
& \leq \frac{N c w}{a b} \exp \left(-\frac{a+b}{a b} w\right) \log \left(1+\frac{a b}{c w}\right) \\
& w \ll 1 \frac{N c}{a b} w \log \frac{1}{w} .
\end{aligned}
$$

Plugging (54) and (55) back into (43) leads to the second part of (8).

\section{REFERENCES}

[1] K. J. R. Liu, A. K. Sadek, W. F. Su, and A. Kwasinski, Cooperative Communications and Networking. Cambridge University Press, 2008.

[2] J. N. Laneman, D. N. C. Tse, and G. W. Wornell, "Cooperative diversity in wireless networks: efficient protocols and outage behavior," IEEE Trans. Inf. Theory, vol. 50, no. 12, pp. 3062-3080, Dec. 2004.

[3] D. Q. Chen and J. N. Laneman, "Modulation and demodulation for cooperative diversity in wireless systems," IEEE Trans. Wireless Commun., vol. 5, no. 7, pp. 1785-1794, July 2006.

[4] W. Guan and K. J. R. Liu, "Performance analysis of two-way relaying with non-coherent differential modulation," IEEE Trans. Wireless Commun., vol. 10, no. 6, pp. 2004-2014, June 2011.

[5] W. Guan and K. J. R. Liu, "Mitigating error propagation for wireless network coding," IEEE Trans. Wireless Commun., vol. 11, no. 10, pp. 3632-3643, Oct. 2012.

[6] W. F. Su, A. K. Sadek, and K. J. R. Liu, "Cooperative communication protocols in wireless networks: performance analysis and optimum power allocation," Wireless Personal Commun., vol. 44, no. 2, pp. 181217, Jan. 2008.

[7] K. G. Seddik, A. K. Sadek, W. F. Su, and K. J. R. Liu, "Outage analysis and optimal power allocation for multinode relay networks," IEEE Signal Process. Lett., vol. 14, no. 6, pp. 377-380, June 2007.

[8] Y. D. Jing and H. Jafarkhani, "Network beamforming using relays with perfect channel information," IEEE Trans. Inf. Theory, vol. 55, no. 6, pp. 2499-2517, June 2009.

[9] M. Kobayashi and X. Mestre, "Impact of CSI on distributed space-time coding in wireless relay networks," IEEE Trans. Wireless Commun., vol. 8, no. 5, pp. 2580-2591, May 2009.

[10] A. S. Ibrahim, A. K. Sadek, W. F. Su, and K. J. R. Liu, "Cooperative communications with relay-selection: when to cooperate and whom to cooperate with?" IEEE Trans. Wireless Commun., vol. 7, no. 7, pp. 2814-2827, July 2008.

[11] Y. D. Jing and B. Hassibi, "Distributed space-time coding in wireless relay networks," IEEE Trans. Wireless Commun., vol. 5, no. 12, pp. 3524-3536, Dec. 2006.

[12] K. G. Seddik, A. K. Sadek, A. S. Ibrahim, and K. J. R. Liu, "Design criteria and performance analysis for distributed space-time coding," IEEE Trans. Veh. Technol., vol. 57, no. 4, pp. 2280-2292, July 2008.

[13] X. Giraud, E. Boutillon, and J. C. Belfiore, "Algebraic tools to build modulation schemes for fading channels," IEEE Trans. Inf. Theory, vol. 43, no. 3, pp. 938-952, May 1997.

[14] W. F. Su, Z. Safar, and K. J. R. Liu, "Full-rate full-diversity spacefrequency codes with optimum coding advantage," IEEE Trans. Inf. Theory, vol. 51, no. 1, pp. 229-249, Jan. 2005.

[15] S. Katti, S. Gollakota, and D. Katabi, "Embracing wireless interference: analog network coding," in Proc. 2007 ACM SIGCOMM, pp. 397-408.

[16] Q. Li, S. H. Ting, A. Pandharipande, and Y. Han, "Adaptive two-way relaying and outage analysis," IEEE Trans. Wireless Commun., vol. 8, no. 6, pp. 3288-3299, June 2009.
[17] D. Q. Chen, K. Azarian, and J. N. Laneman, "A case for amplify-forward relaying in the block-fading multiple-access channel," IEEE Trans. Inf. Theory, vol. 54, no. 8, pp. 3728-3733, Aug. 2008.

[18] M. Badr and J. C. Belfiore, "Distributed space time codes for the amplify-and-forward multiple-access relay channel," in Proc. 2008 IEEE Int. Symp. Inf. Theory, pp. 2543-2547.

[19] S. A. Jafar, K. S. Gomadam, and C. C. Huang, "Duality and rate optimization for multiple access and broadcast channels with amplifyand-forward relays," IEEE Trans. Inf. Theory, vol. 53, no. 10, pp. 33503370, Oct. 2007.

[20] Z. G. Ding, T. Ratnarajah, and K. K. Leung, "On the study of network coded AF transmission protocol for wireless multiple access channels," IEEE Trans. Wireless Commun., vol. 8, no. 1, pp. 118-123, Jan. 2009.

[21] K. S. Gomadam and S. A. Jafar, "The effect of noise correlation in amplify-and-forward relay networks," IEEE Trans. Inf. Theory, vol. 55, no. 2, pp. 731-745, Feb. 2009.

[22] S. Berger and A. Wittneben, "Cooperative distributed multiuser MMSE relaying in wireless ad-hoc networks," in Proc. 2005 Asilomar Conf. Signals Syst. Comput., pp. 1072-1076.

[23] E. Koyuncu and H. Jafarkhani, "Distributed beamforming in wireless multiuser relay-interference networks with quantized feedback," IEEE Trans. Inf. Theory, vol. 58, no. 7, pp. 4538-4576, July 2012.

[24] M. Abramovitz and I. A. Stegun, Handbook of Mathematical Functions with Formulas, Graphs, and Mathematical Tables, 9th edition. Dover, 1972.

[25] I. S. Gradshteyn and I. M. Ryzhik, Table of Integrals, Series, and Products, 7th edition. Academic, 2007.

[26] J. Salo, H. M. El-Sallabi, and P. Vainikainen, "Impact of double-rayleigh fading on system performance," in Proc. 2006 Int. Symp. Wireless Pervasive Comput..

[27] M. O. Hasna and M. S. Alouini, "A performance study of dual-hop transmissions with fixed gain relays," IEEE Trans. Wireless Commun., vol. 3, no. 6, pp. 1963-1968, Nov. 2004.

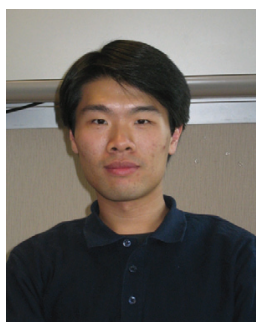

Wei Guan (S'12) received the B.S. in Electrical Engineering and Finance (double degree) in 2006, and M.S. (with highest honor) in Electrical Engineering in 2009, both from Shanghai JiaoTong University, Shanghai, China. Now he is a Ph.D. student in the Department of Electrical and Computer Engineering at University of Maryland, College Park. His current research interests are in the areas of wireless communications and networks, including cooperative communications and network coding. He received the 1st Prize in the 18th National Physics Contest, Shanghai, and the A. James Clark School of Engineering Distinguished Graduate Fellowship from University of Maryland, College Park in 2009.

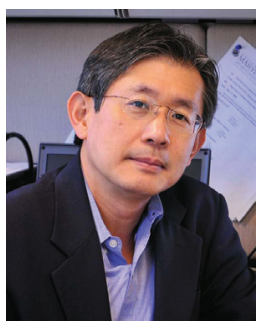

K. J. Ray Liu (F'03) was named a Distinguished Scholar-Teacher of University of Maryland, College Park, in 2007, where he is Christine Kim Eminent Professor of Information Technology. He leads the Maryland Signals and Information Group conducting research encompassing broad areas of signal processing and communications with recent focus on cooperative communications, cognitive networking, social learning and networks, and information forensics and security.

Dr. Liu is the recipient of numerous honors and awards including IEEE Signal Processing Society Technical Achievement Award and Distinguished Lecturer. He also received various teaching and research recognitions from University of Maryland including university-level Invention of the Year Award; and Poole and Kent Senior Faculty Teaching Award and Outstanding Faculty Research Award, both from A. James Clark School of Engineering. An ISI Highly Cited Author in Computer Science, Dr. Liu is a Fellow of IEEE and AAAS.

Dr. Liu is President of IEEE Signal Processing Society where he has served as Vice President - Publications and Board of Governor. He was the Editor-inChief of IEEE Signal Processing Magazine and the founding Editor-in-Chief of EURASIP Journal on Advances in Signal Processing. 